\title{
Nitric Oxide Production and its Contribution to Hepatocyte Proliferation in Normal Juvenile Rats
}

\author{
Naoto INUKAI ${ }^{1}$, Mayuko UCHIDA ${ }^{1)}$, Yoko MIYAZAKI ${ }^{1)}$, Takehito SUZUKI ${ }^{1)}$, Hiroshi YOSHIKAWA ${ }^{2)}$, \\ Kazuaki TANAKA ${ }^{1)}$, Hidetoshi MORITA ${ }^{1,2)}$ and Tatsuya TAKIZAWA ${ }^{1,2) *}$ \\ ${ }^{1)}$ Graduate School of Veterinary Medicine and ${ }^{2)}$ Research Institute of Biosciences, Azabu University, Sagamihara, Kanagawa 252-5201, \\ Japan
}

(Received 9 December 2009/Accepted 10 February 2010/Published online in J-STAGE 24 February 2010)

ABSTRACT. Nitric oxide (NO) has been reported as a key mediator in enhancing hepatocyte proliferation during liver regeneration. Juvenile hepatocytes have a strong ability to proliferate while still in their undifferentiated state but the mechanism of NO production and its contribution to hepatocyte proliferation are not yet fully understood. The present study was designed to investigate NO production in the normal liver and its contribution to hepatocyte proliferation in juvenile rats. Endogenous NO production was evaluated quantitatively using a spin trap followed by electron paramagnetic resonance spectroscopy with the Fe- $N, N$-diethyldithiocarbamate complex as an NOtrapping reagent in the rat liver. NO production in the liver significantly peaked at 3 weeks after birth, but NO synthase (NOS) 3 expression did not change between 2 to 5 weeks after birth, while NOS 1 and NOS 2 mRNA were not detected. Hepatocyte proliferation, measured by the incorporation of 5-bromo-2'-deoxyuridine into the DNA, was found to decline significantly when endogenous NO production was inhibited by the administration of the NOS inhibitor $N^{\mathrm{G}}$-nitro-L-arginine methyl ester. These findings indicate that endogenous NO production peaked at 3 weeks after birth and hepatocyte proliferation declined significantly when NO production was inhibited. Thus, this study provides a novel insight into the contribution of NO to hepatic growth and liver maturation in juveniles. KEY WORDS: electron paramagnetic resonance (EPR) spectroscopy, hepatocyte, nitric oxide.

Nitric oxide (NO) is known to be a multifunctional mediator, regulating blood pressure, gene expression, apoptosis, and mitogenesis in essentially every organ and tissue $[8,10$, 16]. In mammals, NO is enzymatically synthesized from Larginine by three nitric oxide synthase (NOS) isoforms: NOS 1 (neuronal NOS; nNOS), NOS 2 (inducible NOS; iNOS), and NOS 3 (endothelial NOS; eNOS). In adult rats, the hepatocytes are highly differentiated and rarely proliferate, but they retain the ability of proliferating in response to acute or chronic injury [27]. On the other hand, in juvenile rats, the hepatocytes have high proliferative ability and are still undifferentiated with respect to their functions, which appear sequentially during the postnatal developmental stages $[2,7,12]$. Under normal physiological conditions, NOS 3 is constitutively expressed in the endothelial cells of the adult liver, while NOS 1 and NOS 2 are undetectable $[10,13,22]$. On the other hand, NO-derived NOS 2 is one of the key mediators of liver regeneration [16, 26, 27] wherein NOS 2 is expressed inductively in hepatocytes and kupffer cells [21]. However, endogenous NO production and the physiological role of NO in hepatocyte proliferation has not been investigated in juvenile rats.

To characterize the physiological role of $\mathrm{NO}$, direct evaluation of endogenous NO production in organs and tissues is very important. However, one complication is that endogenously generated NO is a short-lived, gaseous free radical $[11,15]$. In addition, NO reacts with many sub-

\footnotetext{
* Correspondence to: Takizawa, T., Graduate School of Veterinary Medicine, Azabu University, 1-17-71, Fuchinobe, Sagamihara-shi, Kanagawa 252-5201, Japan.

e-mail: takizawa@azabu-u.ac.jp
}

stances such as molecular oxygen and superoxide to generate NO derivatives like nitrogen dioxide, peroxynitrite, and nitrate [28]. To overcome these difficulties, a spin trap followed by electron paramagnetic resonance (EPR) spectroscopy with Fe- $N, N$-diethyldithiocarbamate (Fe-DETC) complex as an NO-trapping reagent was applied and the endogenous NO production was detected in several hepatic injury models such as partial hepatectomised $(\mathrm{PH})$ animals and lipopolysaccharide (LPS)-treated animals [14, 18]. However, the developmental production of NO in the liver under normal physiological conditions and its contribution to hepatocyte proliferation has not yet been studied.

In the present study, EPR spectroscopy, with Fe-DETC complex as an NO-trapping reagent, was used to quantify NO production in the liver of juvenile rats. The contribution of NO to hepatocyte proliferation was evaluated by measuring the incorporation of the thymidine analog 5-bromo-2'deoxyuridine (BrdU) into the DNA.

\section{MATERIALS AND METHODS}

Animals: Wistar rats (Charles River Japan, Tokyo, Japan), were maintained on a commercial diet (CE-2; Clea Japan, Tokyo, Japan) and tap water ad libitum and were kept in a room at a temperature of $22^{\circ} \mathrm{C} \pm 3^{\circ} \mathrm{C}$, with a relative humidity of $55 \% \pm 10 \%$, on a constant $12 \mathrm{~L}$ : $12 \mathrm{D}$ schedule. Female rats were impregnated by male rats as they were placed together overnight. The day on which neonates were born was designated as day 0 . Only male juvenile rats were used for this study. The number of neonates was adjusted to 12 at 1 week after birth, and they were weaned at 3 weeks 
after birth.

Detection and quantification of NO production by EPR spectroscopy: Juvenile rats were used at 2, 3, 4, and 5 weeks after birth. DETC ( $400 \mathrm{mg} / \mathrm{kg}$ body weight) and $\mathrm{FeCl}_{3}(80$ $\mathrm{mg} / \mathrm{kg}$ body weight) were administered subcutaneously, and the rats were sacrificed by cervical dislocation $0.5 \mathrm{hr}$ later. In this study, we used DETC that forms a lipophilic iron complex, which serves as a spin trapping reagent to trap NO in the tissues. To avoid formation of an insoluble Fe-DETC precipitate, $\mathrm{FeCl}_{3}$ and DETC were administered separately. Rats were decapitated under ether anesthesia, blood was drawn directly from the abdominal vein, and the liver was rapidly removed and minced with surgical scissors. The minced liver was transferred to a quartz EPR tube and immediately frozen in liquid nitrogen. A standard sample of NO-Fe-DETC was prepared using an NO donor, 4-methyl2-[hydroxyl-imino]-5-nitro-6-methoxy-3-hexanamide (NOR1; Dojindo, Kumamoto, Japan), and Fe-DETC complex. To verify that the EPR spectrum resulted from the activity of hepatic NOS, a NOS-specific inhibitor, L-NAME (100 mg/kg body weight), was administered subcutaneously to 3-week-old rats at 6,12, and $24 \mathrm{hr}$ before sampling. EPR spectra were recorded with an EPR spectrometer (JESTE3X; JEOL, Tokyo, Japan) under the following conditions: microwave frequency $=9131.60-9100.30 \mathrm{MHz}$, microwave power $=10 \mathrm{~mW}$, magnetic field center $=320.0$ $\mathrm{mT}$, magnetic width $+/-=10.00 \mathrm{mT}$, modulation frequency $=100.00 \mathrm{kHz}$, width $=0.32 \mathrm{mT}$, time constant $=0.3$ sec, sweep time $=4.0 \mathrm{~min}$, amplitude $40-100$. A transparent glass Dewar vessel was attached to the EPR spectrometer to record the spectra at $-196^{\circ} \mathrm{C}$. Manganese oxide $(\mathrm{MnO})$ served as an internal standard; it was inserted into the sample cavity to determine the relative intensity of EPR signals in the opposite phase. The height of the NO-Fe-DETC signal serves as an index of $\mathrm{NO}$ formation [23]; the heights of the NO-Fe-DETC and $\mathrm{MnO}$ signals were measured simultaneously, and the ratio of the signal heights was used to quantify NO production, as previously reported [24]. The $g$ value (spectroscopic splitting constant) was calculated from the resonance frequency of the signal and the value of the magnetic field of the $\mathrm{MnO}$ internal standard.

Detection of the mRNA expression of NOS isoforms by using reverse transcription-polymerase chain reaction (RT$P C R)$ : Total RNA was extracted from the livers of 2, 3, 4, and 5 week-old rats using an RNA extraction kit (Isogen; NPG, Toyama, Japan), as per the manufacturer's instructions. First-strand cDNA was synthesized from total RNA $(1 \mu \mathrm{g})$ using reverse transcriptase (RT; SuperScript III; Gibco BBL, Tokyo) and an oligo $\mathrm{d}(\mathrm{T})_{12}$ primer. PCR amplification of the reverse-transcribed cDNA was carried out with primers specifically designed for

Nos 1 (forward: 5'-TATGAGGAGTGGAAGTGGG-3' and reverse: 5'-GTGAGGAGAAGTGTAGCCG-3', 92 bp);

Nos 2 (forward: 5'-TTCACGACACCCTTCACCACAA3 ' and reverse: 5'-CCATCCTCCTGCCCACTTCCTC-3', $912 \mathrm{bp}$ ) and Nos 3 (forward: 5'-ACCGATTACACGACATTGAG-3' and reverse: 5'-GTCCAAACACTCCACGC-3',
$135 \mathrm{bp}$ ). Glyceraldehyde-3-phosphate dehydrogenase gene (Gapdh) was used as an internal standard. Detection of the NOS isoforms mRNA was carried out as described previously [24]. Briefly, for PCR, $1 \mu l$ of reverse-transcribed cDNA was added to a $20-\mu l$ reaction mixture containing 250 $\mathrm{mM}$ of each of the deoxyribonucleotide triphosphates (dNTPs); next, 1 unit of Taq polymerase (rTaq; Takara, Kyoto, Japan) and $0.2 \mathrm{mM}$ of each primer were added. Thermal cycling conditions were as follows: 20 cycles for Gapdh and 30 cycles for Nos 1, Nos 2, and Nos 3 at $94^{\circ} \mathrm{C}$ for $1 \mathrm{~min}$; at $58^{\circ} \mathrm{C}$ for Nos $1,62^{\circ} \mathrm{C}$ for Nos $2,52^{\circ} \mathrm{C}$ for Nos 3 , and $60^{\circ} \mathrm{C}$ for Gapdh for $1 \mathrm{~min}$; and at $72^{\circ} \mathrm{C}$ for $1 \mathrm{~min}$ (GeneAmp 2400; Perkin Elmer Japan, Yokohama, Japan). The PCR products were separated on a $2.0 \%$ agarose gel and stained with SYBR Green I (Takara, Kyoto, Japan). Detection of rat Nos 1, Nos 2, Nos 3, and Gapdh was performed by scanning the gels with a Fluoro-Image Analyzer (FLA2000; Fujifilm, Tokyo).

Detection of NOS 3 protein by western blotting: NOS 3 and $\beta$-actin protein expression levels were analyzed by western blot using antibodies against NOS 3 and $\beta$-actin. Three lobes of the liver were obtained from the juvenile rats at 2, 3, 4, and 5 weeks after birth under the ether anesthesia. The livers were homogenized in cold lysis buffer $(1 \mathrm{mM}$ EDTA, $1 \mathrm{mM}$ EGTA, 1\% polyoxyethylene octylphenyl ether, $0.1 \mu \mathrm{M}$ PMSF, $2.5 \mathrm{mM}$ sodium pyrophosphate, $1 \mathrm{mM}$ $\beta$-glycerophosphate, $1 \mathrm{mM} \mathrm{Na} \mathrm{VO}_{4}, 1 \mu \mathrm{g} / \mathrm{m} l$ leupeptin, and $50 \mathrm{mM}$ Tris- $\mathrm{HCl}(\mathrm{pH}$ 7.6)). The homogenate was centrifuged at $15,000 \mathrm{rpm}$ for $40 \mathrm{~min}$ at $4^{\circ} \mathrm{C}$, and the supernatant was stored at $-80^{\circ} \mathrm{C}$ until analysis.

Total protein concentrations of the supernatants were assessed using the $\mathrm{BCA}^{\mathrm{TM}}$ Protein Assay kit (Pierce, Rockford, U.S.A.). Supernatants containing $20 \mu \mathrm{g}$ of total protein were boiled at $95^{\circ} \mathrm{C}$ for $5 \mathrm{~min}$ in Tris SDS with $\beta$ mercaptoethanol sample buffer (Daiichi Pure Chemicals, Tokyo, Japan), and each protein was separated on a $7.5 \%$ SDS-polyacrylamide gel. The proteins were transferred by electroblotting to a polyvinylidene difluoride membrane. After washing with tris-buffered saline containing $0.1 \%$ Tween 20 (TBS-T), the membrane was incubated with primary antibodies against NOS 3 (Santa Cruz Biotechnology, Santa Cruz, U.S.A.) or with primary antibodies against $\beta$ actin (Sigma, Saint Louis, U.S.A.) at a dilution of 1:1,000 or $1: 2,000$, respectively, for $60 \mathrm{~min}$. Following the wash with TBS-T, the membrane was incubated with horseradish peroxidase-conjugated secondary antibody (Jackson ImmunoResearch, West Grove, U.S.A.) for $60 \mathrm{~min}$. After the final wash, the blot was analyzed by the chemifluorescence method with a fluoroimage analyzer (FLA-2000; Fujifilm, Tokyo, Japan) using ECL Plus Western Blotting Detection Reagents (GE Healthcare, Little Chalfont, UK). Quantification of the western blot signal intensity was performed using an analysis program (Mac Bas; Fujifilm, Tokyo, Japan).

Immunohistochemical detection of hepatocyte proliferation by BrdU-labeling: The rats at 3 weeks after birth, when the NO production peaked, were intraperitoneally administered with $N^{\mathrm{G}}$-nitro-L-arginine methyl ester (L-NAME; 
Wako Pure Chemical, Osaka, Japan, $100 \mathrm{mg} / \mathrm{kg}$ body weight) $12 \mathrm{hr}$ before sampling, and with BrdU (BrdU; Sigma, Saint Louis, U.S.A., $100 \mathrm{mg} / \mathrm{kg}$ body weight) $1 \mathrm{hr}$ before sampling. The rats were sacrificed under ether anesthesia, and the 3 dissected lobes of each liver were fixed in $10 \%$ phosphate buffered formalin. The fixed lobes were embedded in paraffin and sectioned at $5 \mu \mathrm{m}$. After deparaffinization and dehydration in a graded series of ethanol, these sections were incubated for $20 \mathrm{~min}$ in $2 \mathrm{~N}$ hydrochloric acid, then immersed for $30 \mathrm{~min}$ in phosphate buffered saline (PBS) containing $0.1 \%$ trypsin at $37^{\circ} \mathrm{C}$. This was followed by suppression of endogenous peroxidase activity with a 30 min incubation in methanol containing $0.3 \% \mathrm{H}_{2} \mathrm{O}_{2}$. BrdUlabeled hepatocytes were immunostained using monoclonal antibody against BrdU (Calbiochem, San Diego, U.S.A.) with Vectastain Elite ABC Mouse IgG Kit (Funakoshi, Tokyo, Japan) and a peroxidase staining DAB kit (Nacalai Tesque, Kyoto, Japan). Negative control slides were incubated with serum substituted for the primary antibody. The sections were then stained with Mayer's hematoxylin. The ratio of BrdU incorporated hepatocytes was evaluated by counting at least 1,500 random hepatocytes. All procedures were performed under the guidance of the Committee for Animal Experimentation, Azabu University.

Statistical analysis: Data are expressed as means \pm standard errors (SE). The differences among groups of rats were assessed by ANOVA or the Student's $t$-test. If a significant difference among the groups was observed, then Scheffe's test was used to determine which groups were different. Pvalues less than 0.05 were considered to be statistically sig- nificant.

\section{RESULTS}

Detection of endogenous NO production and developmental changes of NO production in the juvenile rat liver: The EPR spectrum of a standard sample of NO-Fe-DETC in PBS showed the same triplet signal $\left(g_{\perp}=2.038\right)$ described by Suzuki [23] (Fig. 1A). The EPR spectra with Fe-DETC trapping in the liver also showed this triplet signal $(g=2.038)$ (Fig. 1B), which identified the signal as NO-Fe-DETC $\left(g_{\perp}=2.038\right)$ (Fig. 1A). The NO-Fe-DETC $\left(g_{\perp}=2.038\right)$ signal in the liver was superimposed on the signal of $\mathrm{Cu}$-DETC at liquid nitrogen temperature in vivo, as previously reported [23]. The NO-Fe-DETC and MnO signals were measured simultaneously, and the ratio of signal heights was used to quantify NO production (Fig. 1B). Changes in NO production were detected in the liver at 2, 3, 4, and 5 weeks after birth (Fig. 1C). As revealed by EPR analysis, NO production increased significantly at 3 weeks after birth, but low levels of $\mathrm{NO}$ were detected during the other periods.

Effect of L-NAME administration on endogenous NO production in the liver: NO is enzymatically synthesized by NOS in vivo. To investigate whether NO production results from NOS activity in the liver during development, NO production was measured after administration of a NOS inhibitor, L-NAME, at 3 weeks after birth, using a spin trap followed by EPR spectroscopy (Fig. 2). L-NAME was administered 6,12 , and $24 \mathrm{hr}$ before sampling. NO production was completely suppressed 6 and $12 \mathrm{hr}$ after adminis-
(A)

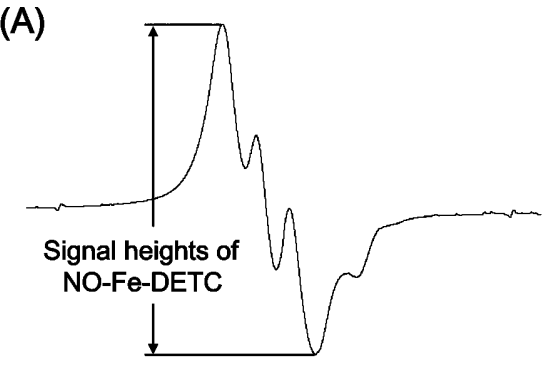

(B)

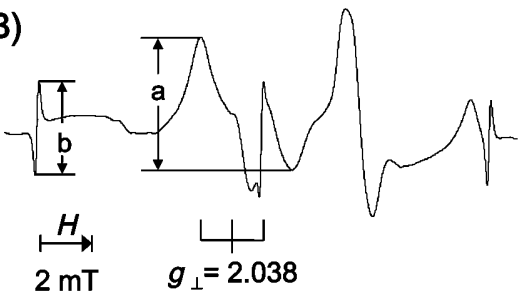

(C)

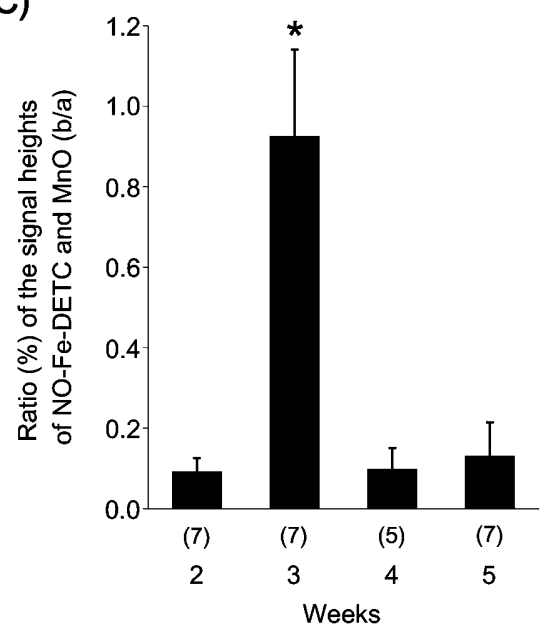

Fig. 1. Detection of endogenous NO production and developmental changes in NO production in the juvenile rat liver. EPR spectra of the liver were recorded at 2, 3, 4, and 5 weeks in rats after subcutaneous administration of DETC solution $(400 \mathrm{mg} / \mathrm{kg})$ and Fe solution $(80 \mathrm{mg} / \mathrm{kg}) 0.5 \mathrm{hr}$ before sampling. (A) EPR spectrum of the standard sample of NO-Fe-DETC complex. (B) EPR spectrum of an NO-Fe-DETC complex detected in the rat liver at 3 weeks. The NO-Fe-DETC (a) and $\mathrm{MnO}$ (b) signal heights were simultaneously analyzed to calculate NO production. (C) Developmental changes in NO production in the juvenile rat liver (means $\pm \mathrm{SE}$ ). The number of animals sampled is shown in parenthesis. ${ }^{*} \mathrm{P}<0.05 ; 3$-week-old rats vs. all other rats. 
(A)

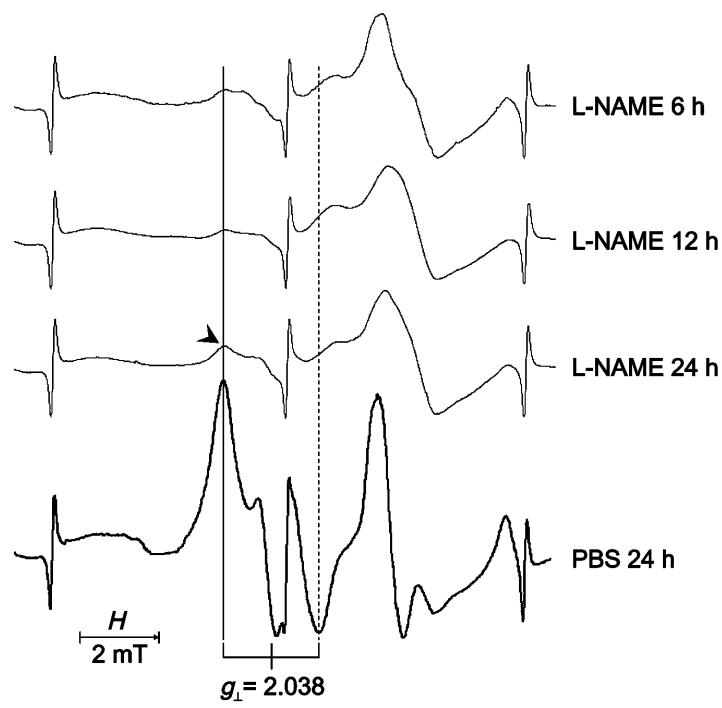

Fig. 2. The suppression of NO production by the NOS inhibitor L-NAME $(100 \mathrm{mg} / \mathrm{kg})$ in the liver at 3 weeks. The tops and bottoms of the NO-Fe-DETC signals are indicated by a solid line and broken line, respectively. L-NAME was administered intraperitoneally 6 (A), 12 (B), and 24 (C) hr before sampling. Phosphate buffered saline (PBS) (D) was used as the vehicle for $\mathrm{L}-$ NAME. Note, only slight NO production (arrow head) was seen in the L-NAME samples treated 24 (C) hr before sampling.

tration of L-NAME (Fig. 2A, B). In the rat liver, $24 \mathrm{hr}$ after administration of L-NAME (Fig. 2C), only slight NO production was observed. The EPR spectrum in the absence of L-NAME (Fig. 2D) showed the typical NO-Fe-DETC signal (Fig. 1B).

Detection of NOS isoform mRNAs in the liver: To examine the expression of the NOS isoforms responsible for NO production in the liver, RT-PCR analysis was performed using total RNA extracted from the liver. NOS 3 mRNA was consistently expressed in the livers of 2, 3, 4, and 5 week-old rats, but NOS 1 and NOS 2 mRNA were undetected during these periods (Fig. 3).

NOS expression in the liver: To investigate the contribution of NOS 3 to NO production in the liver during development, NOS 3 expression was measured at 2, 3, 4, and 5 weeks after birth. The NOS 3 expression level remained unchanged between 2 and 5 weeks after birth (Fig. 4B).

Suppression of hepatocyte proliferation by L-NAME administration: To investigate whether NO production contributes to hepatocyte proliferation in normal juvenile rats, hepatocyte uptake of the thymidine analogue, BrdU, was evaluated as the index of proliferation. The ratio of BrdUlabeled hepatocytes to total hepatocytes was determined in the presence or absence of L-NAME in rats at 3 weeks after birth (Fig. 5). BrDU-incorporated hepatocytes were frequently observed in the control group. No staining was seen in negative control slides in which serum was substituted for the primary antibody (data not shown). The hepatocyte proliferation rate in the presence of L-NAME significantly

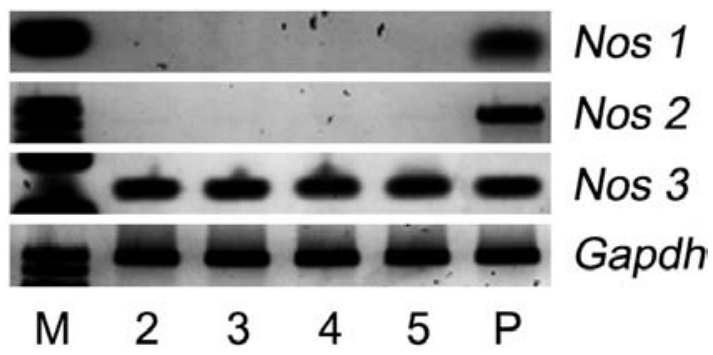

Fig. 3. Developmental changes in the NOS 1, 2, and 3 mRNA in the liver of rats at 2, 3, 4, and 5 weeks of age. Representative separation on a $2.0 \%$ agarose gel of the RT-PCR products amplified from total RNA liver extracts. The expected product sizes were $92 \mathrm{bp}$ for Nos 1 (A), 912 bp for Nos 2 (B), 135 bp for Nos-3 (C), and 983 bp for Gapdh (D). M, DNA size markers. P, positive controls from the brain and placenta for Nos 1 and Nos 2, respectively.

(A)

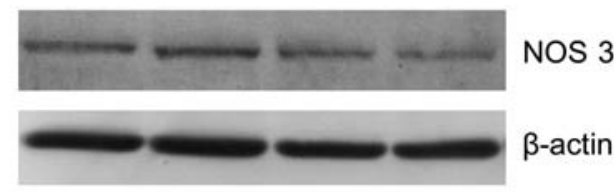

(B)

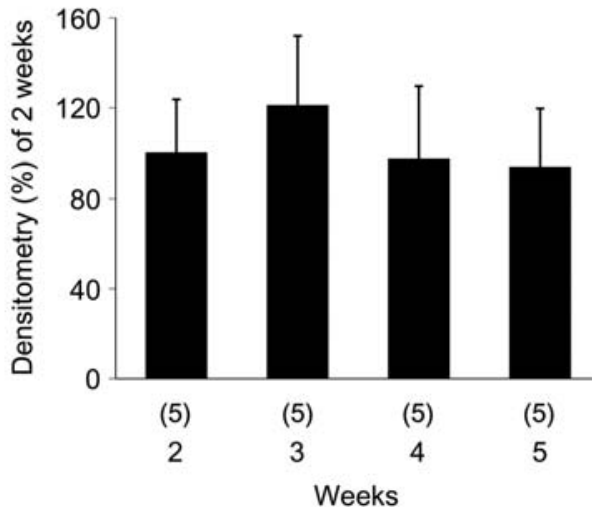

Fig. 4. Developmental changes in NOS 3 expression in the liver of rats at 2, 3, 4, and 5 weeks. Proteins ( $20 \mu \mathrm{g} / \mathrm{lane})$ were separated in $7.5 \%$ gel and analyzed by western blot using antibodies against NOS 3 and $\beta$-actin. (A) Representative blots of NOS 3 and $\beta$-actin are shown. (B) Changes in NOS 3 were expressed as a comparison to samples from 2 -week-old rats (means $\pm \mathrm{SE}$ ). The number of animals is shown in parenthesis.

decreased to one-sixth that of the control group $(\mathrm{P}<0.05)$.

\section{DISCUSSION}

In the present study, NO production in the juvenile rat liver was directly demonstrated by a spin trap followed by EPR spectroscopy. The triplet EPR signal $\left(g_{\perp}=2.038\right)$ observed in the present study was identical to the EPR spectrum of standard NO-Fe-DETC, as previously reported [23]. Moreover, quantification of NO production in the liver revealed that $\mathrm{NO}$ production peaked significantly at 3 weeks 


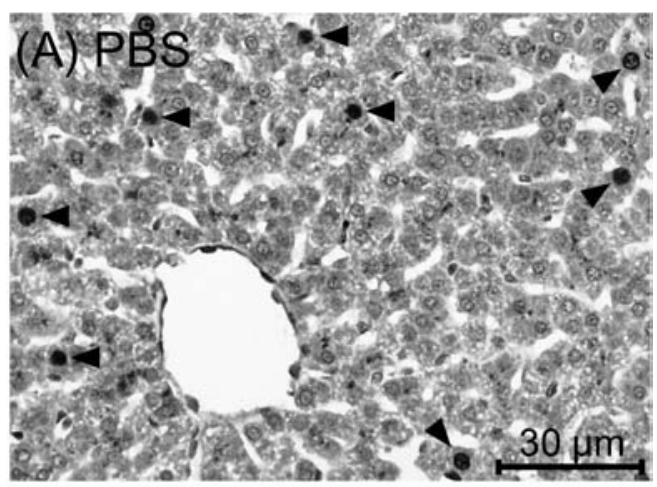

(C)
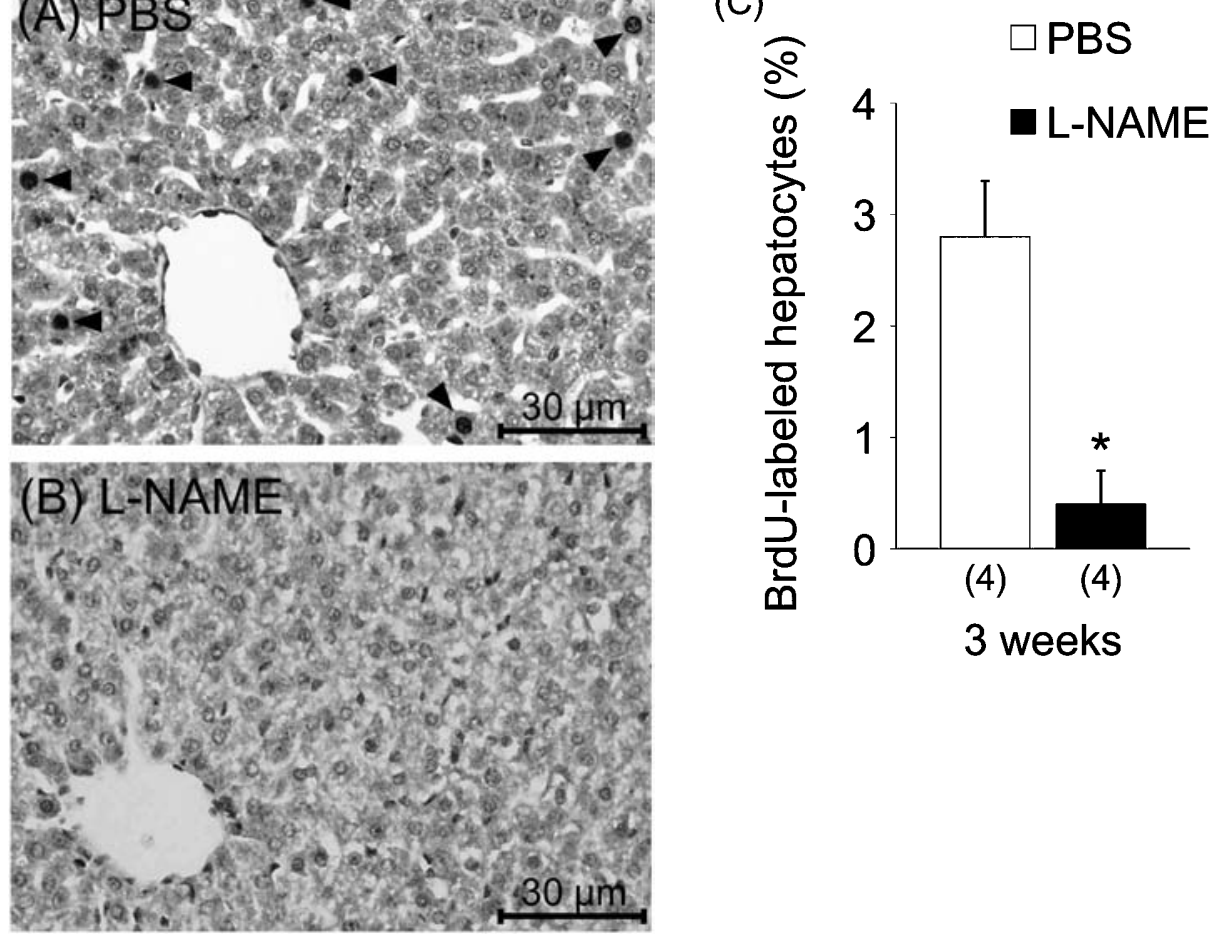

Fig. 5. Suppression of hepatocyte proliferation by the administration of L-NAME. L-NAME $(100 \mathrm{mg} /$ $\mathrm{kg})$ and $\mathrm{BrdU}(100 \mathrm{mg} / \mathrm{kg})$ were intraperitoneally administered 12 and $1 \mathrm{hr}$ before sampling, respectively. Representative sections of liver-administered phosphate buffered saline (PBS) (A) and LNAME (B) from 3-week-old rats are shown. Note BrdU-positive hepatocyte (arrow heads). Slides that were incubated with serum substituted for the primary antibody showed no staining (data not shown). (C) The hepatocyte proliferation rate is shown as the ratio of BrdU-labeled hepatocytes to total hepatocytes, from a sample size of at least 1,500 hepatocytes (means $\pm \mathrm{SE}$ ). The number of animals is shown in parenthesis. ${ }^{*} \mathrm{P}<0.05$; L-NAME vs. PBS.

after birth. The EPR signal observed in the liver with FeDETC trapping after L-NAME administration showed loss of the triplet signal only, indicating that $\mathrm{NO}$ was exclusively synthesized by hepatic NOS. Meanwhile, NOS 1 and NOS 2 mRNA were not detected in the liver during juvenile stages. Previous report has suggested that NOS 3 is the only NOS isoform expressed in the liver of newborn rats, and its expression continues into adulthood [13]. Thus, it is suggested that the NO production in the liver was derived from NOS 3.

Evaluations of endogenous NO production in biological systems have been performed in liver injury models such as LPS-treated animals and regenerating livers [14, 18, 23]. In injury models, NO is largely produced by NOS 2, and the NO production pattern is similar to that of the NOS 2 gene expression pattern. In contrast, our results showed that NO was produced by NOS 3 , and the NO production pattern did not correlate with the NOS 3 expression pattern. This difference of whether the produced NO comes from NOS 2 or NOS 3 could be caused by NOS 3 activation, such as phosphorylation or increment of intracellular $\mathrm{Ca}^{2+}$ concentration $[1,5,9,17]$. To estimate the contribution of NOS 3 to NO production, it is necessary to investigate NOS 3 phosphorylation, intracellular $\mathrm{Ca}^{2+}$ concentrations, and other factors contributing to NOS 3 activity. The investigation of all of these factors under normal physiological in vivo conditions is difficult. The present study showed that a spin trap followed by EPR spectroscopy must be applied to estimate NO production through NOS 3 under normal physiological conditions. Obolenskaya et al. [14] used methods similar to those reported here and demonstrated that NO production is very low in adult rats. In addition, NOS 3 (eNOS) protein in the liver reaches a maximum 20 days after birth, and these levels persist in adults [13]. These reports were in good agreement with the present study, where minimal NO production was observed in the livers of normal 5-week-old rats, when NOS 3 protein expression is almost at the same level as in adults.

As previously reported, the proliferation of mature hepatocytes and the proliferation and differentiation of progenitor cells, induced by NO, mediates regeneration in the adult rat liver [3, 4]. We demonstrated that the administration of the NOS inhibitor L-NAME inhibits NO production and strongly suppresses hepatocyte proliferation in the liver at 3 
weeks after birth. These results proved that NO promotes both hepatocyte proliferation in juveniles and regeneration of adult liver tissue. NO has been reported as a key mediator of hepatocyte proliferation in acute or chronic injury. In the regenerating liver, NO plays a role in the triggering of the regenerating cascade [19, 20, 25-27]. In addition, in vitro studies have shown that NO sensitizes hepatocytes to hepatic growth factor-induced proliferation by modifying the production of s-adenosylmethionine [6]. The liver at 3 weeks after birth shows significantly high NO production when the organ is in the late maturation stage of development. Liver maturation is caused by an increasing number of differentiated hepatocytes that hyperexpress tryptophan 2,3-dioxygenase (TDO) [12]. Expression of mature hepatocyte markers, such as TDO and cholesterol $7 \alpha$-hydroxylase (CYP7A), is evident at 3 weeks after birth. Simultaneously, NO may cause the increasing number of differentiated hepatocytes to activate the liver regenerating cascade $[19,20$, 25-27]. Further study is required to describe the molecular mechanism of NO underlining hepatocyte proliferation in the juvenile stages.

In conclusion, endogenous NO production was directly detected in the liver of juvenile rats using $\mathrm{NO}$ trapping by Fe-DETC combined with EPR spectroscopy. NO production in the liver peaked at 3 weeks after birth in juvenile rats. The NOS inhibitor L-NAME significantly suppressed hepatocyte proliferation in the liver in 3-week-old rats. Our findings suggest that NO is synthesized by activated NOS 3 in the liver of rat to maintain hepatocyte proliferation, and $\mathrm{NO}$ may contribute to liver maturation by increasing the number of differentiated hepatocytes at 3 weeks after birth in rats.

ACKNOWLEDGMENTS. We thank Dr. Daisuke Kiga and Dr. Masahiko Uchiyama for helpful discussions. This work was supported in part by a Grant-in-Aid for Matching Fund Subsidy for Private Universities from The Promotion and Mutual Aid Corporation for Private Schools of Japan.

\section{REFERENCES}

1. Dimmeler, S., Fleming, I., Fisslthaler, B., Hermann, C., Busse, R. and Zeiher, A. M. 1999. Activation of nitric oxide synthase in endothelial cells by Akt-dependent phosphorylation. Nature 399: 601-605.

2. Enomoto, N., Kitamura, T., Hirose, M., Ikejima, K., Watanabe, S. and Sato, N. 1998. Differential $\mathrm{Ca}^{2+}$ signaling in neonatal and adult rat hepatocytes doublets. J. Hepatol. 28: 221-230.

3. Fausto, N. and Campbell, J. S. 2003. The role of hepatocytes and oval cells in liver regeneration and repopulation. Mech. Dev. 120: 117-130.

4. Fausto, N., Campbell, J. S. and Riehle, K. J. 2006. Liver regeneration. Hepatology 43: S45-S53.

5. Fleming, I., Fisslthaler, B., Dimmeler, S., Kemp, B. E. and Busse, R. 2001. Phosphorylation of Thr (495) regulates $\mathrm{Ca}(2+) /$ calmodulin-dependent endothelial nitric oxide synthase activity. Circ. Res. 88: E68-E75.

6. García-Trevijano, E. R., Martínez-Chantar, M. L., Latasa. M. U., Mato, J. M. and Avila, M. A. 2002. NO sensitizes rat hepatocytes to proliferation by modifying s-adenosylmethionine levels. Gastroenterology 122: 1355-1363.

7. Greengard, O. 1970. Enzymic differentiation in mammalian tissues. Essays Biochem. 7: 159-205.

8. López-Farré, A., Rodríguez-Feo, J. A., Sánchez, de, Miguel, L., Rico, L. and Casado, S. 1998. Role of nitric oxide in the control of apoptosis in the microvasculature. Int. J. Biochem. Cell. Biol. 30: 1095-1106.

9. Makondo, K., Kimura, K., Kitamura, N., Kitamura, T., Yamaji, D., Jung, B. D. and Saito, M. 2003. Hepatocyte growth factor activates endothelial nitric oxide synthase by $\mathrm{Ca}(2+)$ - and phosphoinositide 3-kinase/Akt-dependent phosphorylation in aortic endothelial cells. Biochem. J. 374: 63-69.

10. Mittal, M. K., Gupta, T. K., Lee, F. Y., Sieber, C. C. and Groszmann, R. J. 1994. Nitric oxide modulates hepatic vascular tone in normal rat liver. Am. J. Physiol. 267: G416-G422.

11. Moncada, S., Palmer, R. M. J. and Higgs, E. A. 1991. Nitric oxide: physiology, pathophysiology and pharmacology. Pharmacol. Rev. 43: 109-142.

12. Nagao, M., Nakamura, T. and Ichihara, A. 1986. Developmental control of gene expression of tryptophan 2,3-dioxygenase in neonatal rat liver. Biochim. Biophys. Acta 867: 179-186.

13. Nowicki, M. J., Shi, D., Cai, Z., Bishop, P. R. and May, W. L. 2003. Developmental expression of endothelial nitric oxide synthase (eNOS) in the rat liver. Pediatr. Res. 54: 732-738.

14. Obolenskaya, M. Y., Vanin, A. F., Mordvintcev, P. I., Mülsch, A. and Decker, K. 1994. EPR evidence of nitric oxide production by the regenerating rat liver. Biochem. Biophys. Res. Commun. 202: 571-576.

15. Palmer, R. M. J., Ferrige, A. G. and Moncada, S. 1987. Nitric oxide release accounts for the biological activity of endothelium-derived relaxing factor. Nature 327: 524-526.

16. Rai, R. M., Lee, F. Y. J., Rosen, A., Yang, S. Q., Lin, H. Z., Koteish, A., Liew, F. Y., Zaragoza, C., Lowenstein, C. and Diehl, A. M. 1998. Impaired liver regeneration in inducible nitric oxide synthase-deficient mice. Proc. Natl. Acad. Sci. U.S.A. 95: 13829-13834.

17. Repetto, S., Salani, B., Maggi, D. and Cordera, R. 2005. Insulin and IGF-I phosphorylate eNOS in HUVECs by a caveolin-1 dependent mechanism. Biochem. Biophys. Res. Commun. 337: 849-852.

18. Ronco, M. T., Alvarez, M. L., Monti, J. A., Carrillo, M. C., Pisani, G. B., Lugano, M. C. and Carnovale, C. E. 2004. Role of nitric oxide increase on induced programmed cell death during early stages of rat liver regeneration. Biochim. Biophys. Acta 1690: 70-76.

19. Schoen, J. M., Wang, H. H., Minuk, G. Y. and Lautt, W. W. 2001. Shear stress-induced nitric oxide release triggers the liver regeneration cascade. Nitric Oxide 5: 453-464.

20. Schoen-Smith, J. M. and Lautt, W. W. 2005. The role of prostaglandins in triggering the liver regeneration cascade. Nitric Oxide 13: 111-117.

21. Serracino-Inglott, F., Virlos, L. T., Habib, N. A., Williamson, R. C. N. and Mathie, R. T. 2003. Differential nitric oxide synthase expression during hepatic ischemia-reperfusion. $\mathrm{Am}$. J. Surg. 185: 589-595.

22. Shah, V., Haddad, F. G., Garcia-Cardena, G., Frangos, J. A., Mennone, A., Groszmann, R. J. and Sessa, W. C. 1997. Liver sinusoidal endothelial cells are responsible for nitric oxide modulation of resistance in the hepatic sinusoids. J. Clin. Invest. 100: 2923-2930.

23. Suzuki, Y., Fujii, S., Numagami, Y., Tominaga, T., Yoshimoto, T. and Yoshimura, T. 1998. In vivo nitric oxide detection in the septic rat brain by electron paramagnetic resonance. Free 
Radic. Res. 28: 293-299.

24. Takizawa, T., Yoshikawa, H., Yamada, M. and Morita, H. 2002. Expression of nitric oxide synthase isoforms and detection of nitric oxide in rat placenta. Am. J. Physiol. Cell Physiol. 282: C762-C767.

25. Tuncyurek, P., Yenisey, C., Doger, F. K., Soyder, A., Bicakci, T. and Cevikel, M. H. 2006. Nitric oxide as an independent regulatory factor in regenerating rat liver. Acta Chir. Belg. 106: 581-587.
26. Wang, H. H. and Lautt, W. W. 1998. Evidence of nitric oxide, a flow-dependent factor, being a trigger of liver regeneration in rats. Can. J. Physiol. Pharmacol. 76: 1072-1079.

27. Weglarz, T. C. and Sandgren, E. P. 2000. Timing of hepatocyte entry into DNA synthesis after partial hepatectomy is cell autonomous. Proc. Natl. Acad. Sci. U.S.A. 97: 12595-12600.

28. Yoshimura, T. 1996. Endogenous nitric oxide, its chemical characterization and the high affinity for metal ions and metalloproteins. CVD Grand Round Ser. 3: 1-26. 